\title{
Micro-scale 2D quasi-nanosheets formed by OD nanocrystals: from single to multicomponent building blocks
}

\author{
Yu Chang, Meng Xu, Liu Huang, Rongrong Pan, Jiajia Liu, Jia Liu, Hongpan Rong, \\ Wenxing Chen and Jiatao Zhang
}

\begin{abstract}
Self-assembly of colloidal nanocrystals (NCs) into large-scale superlattices with complex and controllable structures has attracted extensive attention due to their collective properties and promising device applications. Plasmonic NCs are very popular for long-range ordered superstructures by virtue of their collective nanogaps for electromagnetic field enhancement, in particular bulk-scale single-layer assembly. Large-area two-dimensional (2D) quasinanosheets (QNSs) composed of mono-component Au NCs or multi-component Au@ZnS core-shell hetero-nanocrystals (HNCs) were successfully prepared, via careful solvent evaporation-induced interfacial self-assembly. The entire selfassembly process was carried out on the liquid-air surface and mediated simply by tuning the operating temperatures and concentrations of the NCs. Specifically, monolayer and double-layer 2D QNSs in tens of micrometers scale with different stacking models were fabricated by precisely controlling the solvent evaporation rate and colloidal concentration.
\end{abstract}

Keywords: nanocrystals, self-assembly, plasmonic superstructures, 2D nanosheets

\section{INTRODUCTION}

Superstructures with complex compositions and tailored functions can be conveniently produced by the assembly of nanoparticles [1-3]. Colloidal nanocrystals (NCs) have attracted extensive attention because of their unique properties and applications induced by high monodispersity [4-9]. Hence, the self-assembly of these 0-dimensional (0D) building blocks into complex and multidimensional superstructures is the key step to their versatile applications [10]. The $1 \mathrm{D}, 2 \mathrm{D}$ and $3 \mathrm{D}$ superstructures self-assembly come out successively, and especially the 2D superstructures with programmable design into micrometer nanosheets formation have been widely investigated but far from applications [11-14], in particular the self-assembly plane endowing monolayer NCs building blocks. These kinds of 2D nanosheets are eagerly expected for good performances because of their large surface-to-volume ratio $[15,16]$. Moreover, the flexible assembly of new structured building blocks into $2 \mathrm{D}$ nanosheets is still critical for extensive exploration of nanotechnology.

It is well explored that monodisperse NCs can be spontaneously assembled into ordered structures by the self-volatilization of solvents. Drop casting, spin coating, dip coating, spray coating and blade coating are common self-assembly methods based on solvent evaporation $[17,18]$, which are hindered by isolation from substrates. Solvent evaporation-induced self-assembly proceeding on an immiscible liquid surface can overcome the substrate restrictions. Besides, the self-assembly at the mixed solvent interface is applicable to NCs with a variety of sizes, shapes, structures and components, offering a facile and generalized route for fabricating film-scale 2D nanosheets $[19,20]$. The stacking modes would be affected by the characters of NCs, such as size, shape and component, interactions including van der Waals force, coulombic force and entropic force, in addition to external environmental factors including temperature, humidity and pressure [21-28].

The self-assembly of plasmonic NCs into large-area 2D stacking modes is very important due to their collective electromagnetic field enhancements enabled by their nanogaps [29-31]. On account of the localized surface plasmon resonance (LSPR) of Au NCs, their large-scale

Beijing Key Laboratory of Construction-Tailorable Advanced Functional Materials and Green Applications, Experimental Center of Advanced Materials, School of Materials Science \& Engineering, Beijing Institute of Technology, Beijing 100081, China

* Corresponding author (email: zhangjt@bit.edu.cn) 
assemblies have been applied in plasmonic imaging and catalysis [32-34]. Among these Au NCs, Au@semiconductor core-shell hetero-nanocrystals (HNCs) with plasmon-exciton coupling and optimal dielectric environment are greatly expected for applications $[21,35]$. We have synthesized plasmonic metal@semiconductor HNCs via a reverse cation exchange-enabled nonepitaxial growth strategy [36]. By this way, the as-formed plasmonic HNCs have been found with excellent plasmon enhancement and photocatalysis applications [37,38].

In this work, we chose both plasmonic Au NCs and Au@ZnS core-shell HNCs as building blocks to explore their large-scale 2D self-assemblies. With the solvent evaporation-induced interfacial self-assembly we formed 2D quasi-nanosheets (QNSs) on liquid-air surface which could be easily transferred onto different substrates. Microscale single-layer and double-layer QNSs were formed controllably. These film-like 2D QNSs are uniform and can be easily transferred to other substrates without being destroyed. Besides, the flexible stacking mode could be regulated by adjusting the evaporation temperature.

\section{EXPERIMENTAL SECTION}

\section{Chemicals}

Tetrachloroauric(III) acid hydrate $\left(\mathrm{HAuCl}_{4} \cdot 4 \mathrm{H}_{2} \mathrm{O}\right)$, silver nitrate $\left(\mathrm{AgNO}_{3}, \geq 99 \%\right.$, analytical reagent), zinc nitrate hexahydrate $\left[\mathrm{Zn}\left(\mathrm{NO}_{3}\right)_{2} \cdot 6 \mathrm{H}_{2} \mathrm{O}, 99.99 \%\right]$, sublimed sulfur (99.5\%), oleylamine (OAm, approximate $\mathrm{C} 18$ content of $80 \%-90 \%)$, oleic acid (OA, technical grade, $90 \%$ ), and tributylphosphine (TBP, 95\%) were obtained from Aladdin Reagent. Methanol (anhydrous, 99.8\%), ethanol (anhydrous, $\geq 99.5 \%$ ), ethylene glycol (EG) and toluene (anhydrous, 99.8\%) were purchased from Sinopharm Chemical Reagent. Ultra-pure water was obtained from PURELAB Ultra (resistivity of $18 \mathrm{M} \Omega \mathrm{cm}$ ). All chemicals were used as-received without further processing.

\section{Synthesis of monodisperse plasmonic Au NCs}

$\mathrm{Au}$ seeds with a diameter of 5-6 $\mathrm{nm}$ were synthesized according to the previous report [39]. The obtained $\mathrm{Au}$ NCs were washed and re-dispersed in $30 \mathrm{~mL}$ of toluene, and the $\mathrm{Au}$ NCs colloidal suspension in toluene was uniformly dispersed by ultrasound. At the same time, a toluene solution of tetrachloroauric acid hydrate was prepared at a concentration of $4 \mathrm{mg} \mathrm{mL}^{-1} .2 \mathrm{~mL}$ of this solution and $10 \mathrm{~mL}$ of colloidal $\mathrm{Au}$ NCs were mixed uniformly and put into a sealed glass bottle with a capacity of $20 \mathrm{~mL}$. Au NCs with a diameter of $10-12 \mathrm{~nm}$ were achieved after reacting in an oven at $80^{\circ} \mathrm{C}$ for $5 \mathrm{~h}$.

\section{Synthesis of the plasmonic Au@ZnS core-shell HNCs}

The preparation of $\mathrm{Au} @ \mathrm{Ag}$ and $\mathrm{Au} @ \mathrm{Ag}_{2} \mathrm{~S}$ core-shell nanoparticles were according to the previous publications $[40,41]$. The as-prepared $\mathrm{Au} @ \mathrm{Ag}_{2} \mathrm{~S}$ nanoparticles were separated from the solution by centrifugation and redispersed in $10 \mathrm{~mL}$ of toluene. $0.2 \mathrm{~mL}$ of $\mathrm{OA}, 0.1 \mathrm{~mL}$ of $\mathrm{OAm}$ and $0.8 \mathrm{~mL}$ of $\mathrm{Zn}\left(\mathrm{NO}_{3}\right)_{2}$ dissolved in methanol were added into $10 \mathrm{~mL}$ of the $\mathrm{Au} @ \mathrm{Ag}_{2} \mathrm{~S}$ colloidal solution in toluene. $0.05 \mathrm{~mL}$ of TBP was added to the abovementioned mixture after stirring for $5 \mathrm{~min}$, and the entire solution was stirred vigorously for $2 \mathrm{~h}$ at $60^{\circ} \mathrm{C}$ in oil bath. The resulting Au@ZnS HNCs were collected by centrifugation at $5000 \mathrm{r} \mathrm{min}^{-1}$ for $10 \mathrm{~min}$, and re-dispersed in toluene.

\section{Self-assembly of the as-prepared monodisperse NCs}

The monodispersity of the as-prepared Au and Au@ZnS NCs were further improved by gradient centrifugation. A series of $\mathrm{Au}$ or Au@ZnS NCs with different sizes were achieved by adding 3 or $2 \mathrm{~mL}$ of ethanol as the gradient amount, and the final precipitates were re-dispersed in toluene for further self-assembly. Our approach was based on a drying-driven dynamic self-assembly process occurring on the air-liquid surface. EG was used as the supporting substrate on which $30 \mu \mathrm{L}$ of the as-prepared NCs colloidal solution was dropped gently and uniformly. The entire system was placed in a sealed environment (oven) to prevent external interferences. As toluene evaporated in air, the NCs self-assembled into 2D QNSs. By adjusting the concentration of the colloidal NCs, largescale single-layer and double-layer $2 \mathrm{D}$ nanosheets can be obtained. When the concentration was $0.1 \mathrm{mg} \mathrm{mL}^{-1}, \mathrm{Au}$ NCs assembled into monolayer QNSs, and the doublelayer QNS were achieved as the concentration increased to $0.3 \mathrm{mg} \mathrm{mL}^{-1}$. Similarly, single-layer and double-layer structures of Au@ZnS HNCs were obtained at the concentration of 0.2 and $0.5 \mathrm{mg} \mathrm{mL}^{-1}$, respectively. The selfassembly temperature of Au NCs and Au@ZnS HNCs was maintained at $30^{\circ} \mathrm{C}$ in a vacuum oven. Especially, in the self-assembly process of Au@ZnS HNCs, by precisely changing the operating temperature $\left(30\right.$ or $\left.40^{\circ} \mathrm{C}\right)$, the chainlike structure and ring structure were formed. The resulting products could be transferred from the liquid surface to other substrates for further observation.

\section{Characterizations of structure and morphology}

Low-resolution transmission electron microscopy (LRTEM) performed on a JEOL JEM-1200EX instrument at $80 \mathrm{kV}$ and high-resolution transmission electron microscopy (HRTEM) performed on an FEI TecaiG2 F20 S- 
Twin instrument at $200 \mathrm{kV}$ were utilized to characterize the details of morphology and interfacial lattice of the prepared nanoparticles as well as their self-assemblies. Besides, microscopy images were obtained using a LEXT OLS5000 3D laser confocal scanning electron microscope (SEM).

\section{RESULTS AND DISCUSSION}

\section{Micrometer QNSs formed by monodisperse Au NCs}

The TEM image of Au seeds was shown in Fig. S1. As illustrated by LRTEM in Fig. S2, the as-prepared Au NCs showed a tendency to self-assemble. The monodispersity of $\mathrm{Au}$ NCs was further optimized by gradient centrifugation (Fig. S3). In order to achieve the expected results, Au NCs (about $10 \mathrm{~nm}$ ) obtained by gradient centrifugation when $12 \mathrm{~mL}$ of ethanol was added were chosen to self-assemble due to their uniform sizes and shapes. The NCs retain the morphology in the colloidal solution with excellent stability for at least half a year, which are reactive enough to self-assemble into $2 \mathrm{D}$ superstructures. The solvent evaporation-induced interfacial self-assembly is a facile and versatile method to fabricate large-scale 2D superstructures [42,43]. The selfassembly process was observed upon dropwise adding $30 \mu \mathrm{L}$ of $\mathrm{NC}$ suspension in toluene on the surface of EG confined in a glass container $\left(\pi \times 1^{2} \times 2 \mathrm{~cm}^{3}\right)$, as depicted in Fig. 1. As toluene gradually evaporated in air, the monodispersed $\mathrm{Au}$ NCs deposited on the surface of EG and arranged into ordered arrays. Thus, the micro-scale
2D "nanosheets" (Fig. 2a) were formed on the liquid surface, confirmed by the TEM image (Fig. 2b). The enlarged TEM images further confirmed that the film was composed of regularly and densely packed Au NCs (Fig. 2c). This novel 2D superstructure was denoted as 2D QNSs. These well-defined 2D QNS with lateral sizes up to several micrometers even tens of micrometers perform an excellent stability on substrates, which can keep their morphology and properties the same for one year.

Several factors, such as temperature, humidity and interface types, have great influences on the process of solvent evaporation-induced interfacial self-assembly [44-46]. By precisely controlling the solvent evaporation rate and the concentration of colloidal NCs, monolayer and double-layer 2D QNSs were successfully fabricated on the air-liquid interface (Fig. 2c, d) and their stacking patterns were shown in Fig. 2e, f. In the $2 \mathrm{D}$ monolayer QNS, Au NCs were spontaneously arranged into the close-packed mode by virtue of their excellent monodispersity. A local position of QNS manufactured by selfassembly of colloidal Au NCs observed by TEM (the inserted picture in Fig. 2c) showed a typical 2D structure in which the Au NCs adopted a pseudohexagonal closepacked arrangement. In the same plane, each Au NC was uniformly and closely surrounded by the other six Au NCs, and a gap was formed among every three adjacent $\mathrm{Au}$ NCs. A characteristic feature of the pseudohexagonal array was that the Au NCs were not in direct contact with each other; they were separated by a region that did not exhibit any diffraction contrast, typically about $2.2 \mathrm{~nm}$,

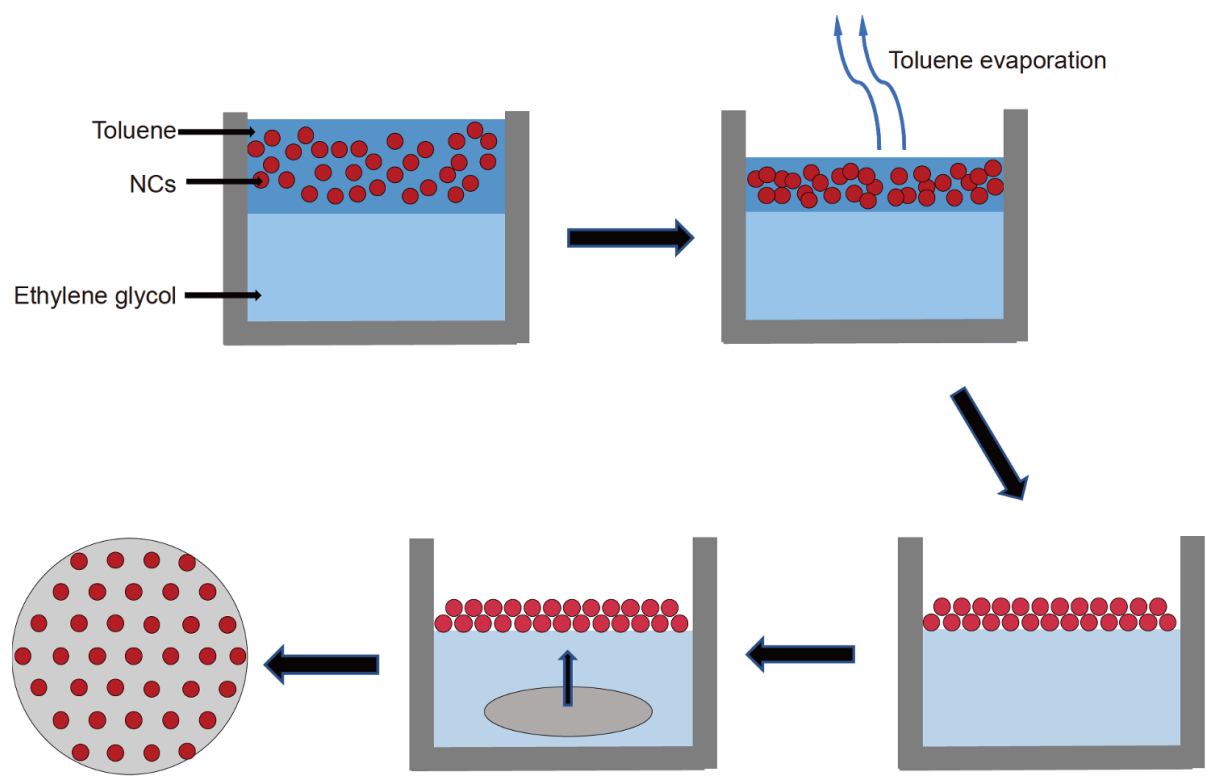

Figure 1 Schematic illustration of the solvent evaporation-induced interfacial self-assembly. 

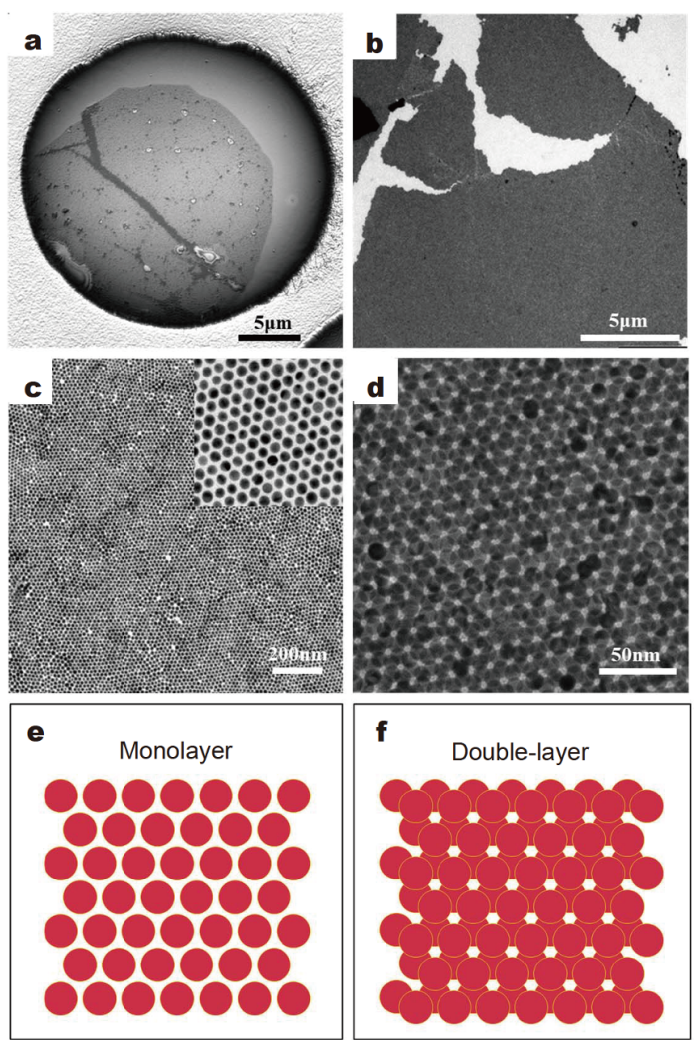

Figure 2 2D QNS formed by the self-assembly of Au NCs. (a, b) Microscopy image and TEM image of the as-prepared 2D Au NC QNS. (c-f) TEM images of monolayer and double-layer QNS and their stacking models.

consistent with the length of the C18 organic surface capping ligands [47]. The double-layer 2D QNS were fabricated by increasing the concentration of colloidal NCs. From the double-layer superlattice films of Au NCs displayed in Fig. 2d, the feature of individual Au NC could be clearly identified. As it was shown in the TEM picture, each $\mathrm{Au} \mathrm{NC}$ in the upper layer occupied the large gap formed by every three adjacent Au NCs in the lower layer, exactly showing how this structure formed. These 2D QNSs formed from Au NCs, including monolayer and double-layer films, had membrane areas of up to the micron scale but the thickness was only several nanometers.

\section{Microscale QNS formed by monodisperse Au@ZnS HNCs} Multi-component HNCs are considered to have collective properties of individual components and are anticipated to provide a designable way to obtain novel materials [48]. Moreover, a previous study of our group about selfassembly of HNCs proved that long-range ordered packing of Au@semiconductor HNCs was propitious for enhancing plasmon-exciton coupling, which in turn could improve their photoelectrically related properties [21]. In the past decades, the self-assembly of simple structure NCs has been investigated widely and detailedly $[10,49,50]$, but few achievements have been made on $2 \mathrm{D}$ nanosheets formed by the self-assembly of core-shell HNCs because of their complex components and unique structures. As a result, their applications are limited by the fact that the formation and manipulation of largescale 2D QNSs remain a major challenge. Herein, with the similar strategy described above, we achieved largescale 2D QNSs of monodisperse Au@ZnS HNCs. TEM images and UV-vis spectra of the Au@ZnS HNCs (core $10 \mathrm{~nm}$, shell $3 \mathrm{~nm}$ ) prepared by non-epitaxial growth strategy and ion exchange method were shown in Fig. S4, in which the HNCs with well-defined structures showed an obvious tendency to self-assemble. The energy-dispersive X-ray spectroscopy (EDS) elemental mapping results manifested the core-shell structure of Au@ZnS HNCs (Fig. S5). Through accurate regulation of the operating temperature (here $30^{\circ} \mathrm{C}$ ) of toluene and the concentration of colloidal Au@ZnS HNCs solution, monolayer and double-layer flexible membranes were formed on the liquid substrate which can be easily transferred to the carbon-coated TEM grids for further characterization, with the size up to tens of micrometers, and could be easily observed under an optical micro-
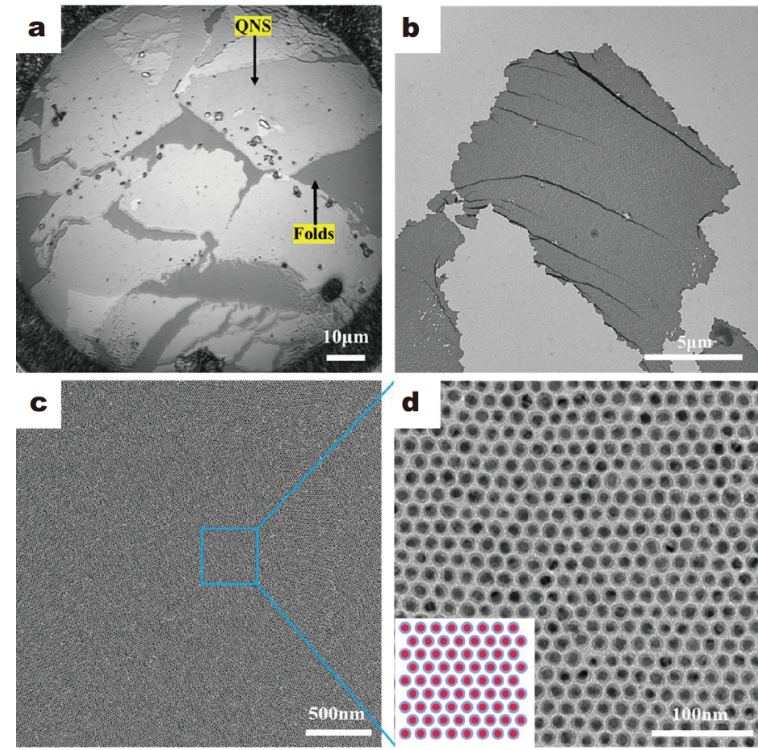

Figure 3 2D QNS formed by the self-assembly of Au@ZnS HNCs. (a, b) Microscope image and TEM image. (c, d) TEM images of monolayer QNS and its stacking model. 
scope. Fig. 3a, b showed the whole film and its folds. The film was continuous and uniform in thickness, as shown in Fig. 3c. For the monolayer nanosheets, Au@ZnS HNCs closely arranged into hexagonal close packing pattern (Fig. 3d), in the same way with that Au NCs accumulated. Each individual Au@ZnS HNC was surrounded by six other HNCs in the same plane, with well-defined structures of core and shell.

The double-layer QNSs of Au@ZnS HNCs were formed for the colloidal NCs with high concentration. The operating temperature is a convenient and important tool to direct self-assembly of NCs toward desired structures because of its ability to tailor relative weights of the interparticle interactions and free volume entropy during the formation of superstructure. In those double-layer QNSs, by precisely controlling the operating temperature $\left(40,30^{\circ} \mathrm{C}\right)$, two different types of non-close-packed (ncp) complex structures were successfully formed on the liquid-air interface, as shown in Fig. 4a (chainlike structure) and Fig. 4c (ring structure). The chainlike structure was achieved at a temperature of $40^{\circ} \mathrm{C}$. In Fig. 4a, Au@ZnS HNCs were arranged in chains with three different directions as straight lines in projection. Interestingly, these three directions rotated by $60^{\circ}$ with respect to each other exactly corresponded to the three different 2fold saddle sites (labeled A, B and C) shown in Fig. 4b. This unique structure was a consequence of occupancies of the 2 -fold saddle sites between two HNCs in the under layer rather than the 3 -fold hollow sites formed by the adjacent three HNCs. In order to understand this phenomenon, Fink et al. [51] proposed that a strong shortrange electrostatic dipolar led to the favorite of 2 -fold saddle sites due to the reduced number of surface dipoles in the 2 -fold sites on the surfaces of the nearest neighbor clusters.

The ring structure shown in Fig. $4 \mathrm{c}$ often observed in bilayer superstructures was obtained at $30^{\circ} \mathrm{C}$. In our study, the diameter of each single ring was about $52 \mathrm{~nm}$. Fig. $4 \mathrm{~d}$ shows that the rings were simply closed chains and each layer of the 2D QNS still kept the ordered pattern. Thus, we can get a conclusion that this ring structure is a result of occupancies of 3-fold saddle sites.

Occasionally, the chainlike structure and ring structure can be observed simultaneously in bilayer QNS (Fig. S6). Murray's group [44] suggested that this was presumably caused by the local variations of colloidal NC concentration during the growth of QNSs. Unlike most domains which were usually spatially separated, there was a smooth transition between these two different domains. These ncp structures defied traditional expectations of
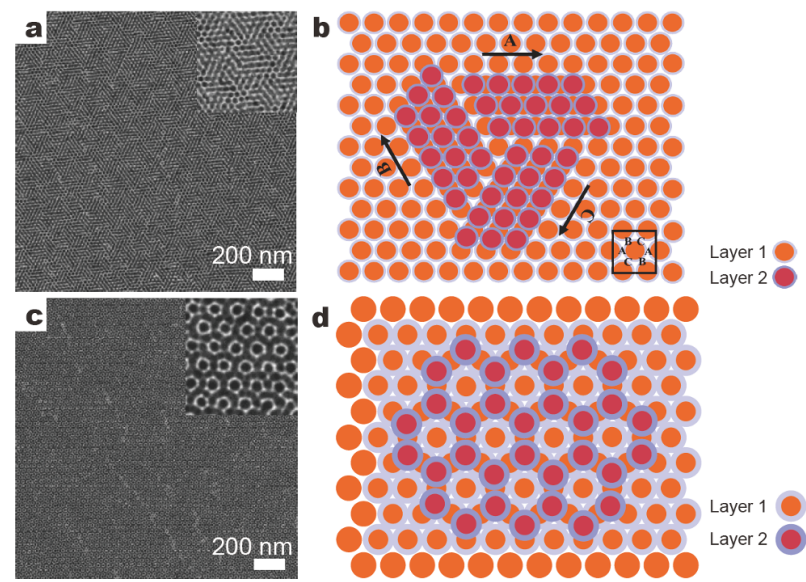

Figure 4 Structures diversities in the double-layer QNS of the Au@ZnS HNCs. (a) TEM image of the double-layer QNS self-assembled by the Au@ZnS HNCs with chainlike structures. (b) Schematic diagram illustrating how three chains with different directions rotated by $60^{\circ}$ with respect to each other can be formed by the upper layer HNCs occupying the 2-fold saddle sites (labeled A, B and C) between two particles in the under layer. (c) TEM image of the double-layer QNS self-assembled by the Au@ZnS HNCs with ring structures. (d) Schematic diagram illustrating how ring structures can be formed by the upper layer HNCs occupying the 3 -fold hollow sites among the three adjacent particles in the under layer.

face-centred-cubic or hexagonal-close-packed arrangements, and showed a possibility of multifarious self-assembly. According to a previous study [52], these longrange-ordered complex phases were stabilized by entropic, steric, dipolar forces and strong local van der Waals interactions between the Au@ZnS HNCs.

\section{CONCLUSIONS}

In summary, we successfully achieved micro-scale 2D QNSs with controllable and long-range ordered superstructures via solvent evaporation-induced liquid-air interfacial self-assembly of single-component $\mathrm{Au}$ NCs or multi-component Au@ZnS core-shell HNCs. The monolayer and double-layer QNS films were prepared by regulating the concentrations of the building blocks. These 2D QNS films could be transferred easily. Stacking diversities realized in the double-layer QNS by further regulation of the assembly temperature. These large-scale and long-range ordered plasmonic QNSs are potential for their plasmonic applications.

\section{Received 19 January 2020; accepted 10 March 2020;} published online 23 April 2020

1 Nagaoka Y, Tan R, Li R, et al. Superstructures generated from truncated tetrahedral quantum dots. Nature, 2018, 561: 378-382 
2 Vogel N, Retsch M, Fustin CA, et al. Advances in colloidal assembly: the design of structure and hierarchy in two and three dimensions. Chem Rev, 2015, 115: 6265-6311

3 Nagaoka Y, Zhu H, Eggert D, et al. Single-component quasicrystalline nanocrystal superlattices through flexible polygon tiling rule. Science, 2018, 362: 1396-1400

4 Zhang M, Magagnosc DJ, Liberal I, et al. High-strength magnetically switchable plasmonic nanorods assembled from a binary nanocrystal mixture. Nat Nanotech, 2017, 12: 228-232

5 Shaw S, Tian X, Silva TF, et al. Selective removal of ligands from colloidal nanocrystal assemblies with non-oxidizing He plasmas. Chem Mater, 2018, 30: 5961-5967

6 Pradhan N, Das Adhikari S, Nag A, et al. Luminescence, plasmonic, and magnetic properties of doped semiconductor nanocrystals. Angew Chem Int Ed, 2017, 56: 7038-7054

7 Wu M, Congreve DN, Wilson MWB, et al. Solid-state infrared-tovisible upconversion sensitized by colloidal nanocrystals. Nat Photon, 2016, 10: 31-34

8 Cai Z, Li F, Xu W, et al. Colloidal CsPbBr ${ }_{3}$ perovskite nanocrystal films as electrochemiluminescence emitters in aqueous solutions. Nano Res, 2018, 11: 1447-1455

9 Chen L, Ji F, Xu Y, et al. High-yield seedless synthesis of triangular gold nanoplates through oxidative etching. Nano Lett, 2014, 14: 7201-7206

10 Boles MA, Engel M, Talapin DV. Self-assembly of colloidal nanocrystals: from intricate structures to functional materials. Chem Rev, 2016, 116: 11220-11289

11 Jiang S, Van Dyk A, Maurice A, et al. Design colloidal particle morphology and self-assembly for coating applications. Chem Soc Rev, 2017, 46: 3792-3807

12 Dai Z, Li Y, Duan G, et al. Phase diagram, design of monolayer binary colloidal crystals, and their fabrication based on ethanolassisted self-assembly at the air/water interface. ACS Nano, 2012, 6: 6706-6716

13 Ji F, Zhong Q, Chen J, et al. High-yield synthesis of Au@Ag right bipyramids and self-assembly into four-leaf-clover-like structures. Part Part Syst Charact, 2018, 35: 1700114

14 van der Stam W, Gantapara AP, Akkerman QA, et al. Self-assembly of colloidal hexagonal bipyramid- and bifrustum-shaped ZnS nanocrystals into two-dimensional superstructures. Nano Lett, 2014, 14: 1032-1037

15 Sun Z, Liao T, Dou Y, et al. Generalized self-assembly of scalable two-dimensional transition metal oxide nanosheets. Nat Commun, 2014, 5: 3813

16 Osada M, Sasaki T. Two-dimensional dielectric nanosheets: novel nanoelectronics from nanocrystal building blocks. Adv Mater, 2012, 24: $210-228$

17 Gaulding EA, Diroll BT, Goodwin ED, et al. Deposition of waferscale single-component and binary nanocrystal superlattice thin films via dip-coating. Adv Mater, 2015, 27: 2846-2851

18 Kim J, Song X, Ji F, et al. Polymorphic assembly from beveled gold triangular nanoprisms. Nano Lett, 2017, 17: 3270-3275

19 Liu D, Cai W, Marin M, et al. Air-liquid interfacial self-assembly of two-dimensional periodic nanostructured arrays. ChemNanoMat, 2019, 5: 1338-1360

20 Talapin DV, Shevchenko EV, Kornowski A, et al. A new approach to crystallization of CdSe nanoparticles into ordered threedimensional superlattices. Adv Mater, 2001, 13: 1868-1871

21 Huang L, Zheng J, Huang L, et al. Controlled synthesis and flexible self-assembly of monodisperse Au@semiconductor core/shell het- ero-nanocrystals into diverse superstructures. Chem Mater, 2017, 29: $2355-2363$

22 Zheng J, Dai B, Liu J, et al. Hierarchical self-assembly of $\mathrm{Cu}_{7} \mathrm{Te}_{5}$ nanorods into superstructures with enhanced SERS performance. ACS Appl Mater Interfaces, 2016, 8: 35426-35434

23 Ye X, Li Y, Dong J, et al. Facile synthesis of ZnS nanobowl arrays and their applications as $2 \mathrm{D}$ photonic crystal sensors. J Mater Chem C, 2013, 1: 6112-6119

24 Cai R, Yang D, Lin KT, et al. Generalized preparation of twodimensional quasi-nanosheets via self-assembly of nanoparticles. J Am Chem Soc, 2019, 141: 1725-1734

25 Liu Q, He P, Yu H, et al. Single molecule-mediated assembly of polyoxometalate single-cluster rings and their three-dimensional superstructures. Sci Adv, 2019, 5: eaax1081

26 Ambrosetti A, Ferri N, DiStasio Jr. RA, et al. Wavelike charge density fluctuations and van der Waals interactions at the nanoscale. Science, 2016, 351: 1171-1176

27 Boles MA, Talapin DV. Self-assembly of tetrahedral CdSe nanocrystals: Effective "patchiness" via anisotropic steric interaction. J Am Chem Soc, 2014, 136: 5868-5871

28 Maestro A, Zaccone A. Nonaffine deformation and tunable yielding of colloidal assemblies at the air-water interface. Nanoscale, 2017, 9: 18343-18351

29 Haidar I, Day A, Martino U, et al. Bottom-up assembly of Au@Ag plasmonic nanocrystals: issues to be addressed to achieve a good SERS substrate. Appl Mater Today, 2019, 15: 462-471

30 Wang $\mathrm{P}, \mathrm{Yu} \mathrm{Q}$, Long $\mathrm{Y}$, et al. Multivalent assembly of ultrasmall nanoparticles: One-, two-, and three-dimensional architectures of 2 nm gold nanoparticles. Nano Res, 2012, 5: 283-291

31 Hunt ST, Milina M, Alba-Rubio AC, et al. Self-assembly of noble metal monolayers on transition metal carbide nanoparticle catalysts. Science, 2016, 352: 974-978

32 Zhang H, Guan C, Song N, et al. Self-assembly of high-index faceted gold nanocrystals to fabricate tunable coupled plasmonic superlattices. Phys Chem Chem Phys, 2018, 20: 3571-3580

33 Gong J, Li G, Tang Z. Self-assembly of noble metal nanocrystals: fabrication, optical property, and application. Nano Today, 2012, 7: 564-585

34 Gómez-Graña S, Fernández-López C, Polavarapu L, et al. Gold nanooctahedra with tunable size and microfluidic-induced $3 \mathrm{D}$ assembly for highly uniform SERS-active supercrystals. Chem Mater, 2015, 27: 8310-8317

35 Tan C, Chen J, Wu XJ, et al. Epitaxial growth of hybrid nanostructures. Nat Rev Mater, 2018, 3: 17089

36 Bai B, Xu M, Li N, et al. Semiconductor nanocrystal engineering by applying thiol- and solvent-coordinated cation exchange kinetics. Angew Chem Int Ed, 2019, 58: 4852-4857

37 Wang $\mathrm{H}$, Gao Y, Liu J, et al. Efficient plasmonic Au/CdSe nanodumbbell for photoelectrochemical hydrogen generation beyond visible region. Adv Energy Mater, 2019, 9: 1803889

38 Liu J, Feng J, Gui J, et al. Metal@semiconductor core-shell nanocrystals with atomically organized interfaces for efficient hot electron-mediated photocatalysis. Nano Energy, 2018, 48: 44-52

39 Zhang J, Tang Y, Lee K, et al. Nonepitaxial growth of hybrid coreshell nanostructures with large lattice mismatches. Science, 2010, 327: $1634-1638$

40 Pan R, Liu J, Li Y, et al. Electronic doping-enabled transition from n- to p-type conductivity over Au@CdS core-shell nanocrystals toward unassisted photoelectrochemical water splitting. J Mater Chem A, 2019, 7: 23038-23045 
41 Cheng X, Liu J, Feng J, et al. Metal@ $\mathrm{I}_{2}-\mathrm{II}-\mathrm{IV}-\mathrm{VI}_{4}$ core-shell nanocrystals: controlled synthesis by aqueous cation exchange for efficient photoelectrochemical hydrogen generation. J Mater Chem A, 2018, 6: 11898-11908

42 Josten E, Wetterskog E, Glavic A, et al. Superlattice growth and rearrangement during evaporation-induced nanoparticle self-assembly. Sci Rep, 2017, 7: 2802

43 Wang H, Hood ZD, Xia Y, et al. Fabrication of ultrathin solid electrolyte membranes of $\beta-\mathrm{Li}_{3} \mathrm{PS}_{4}$ nanoflakes by evaporation-induced self-assembly for all-solid-state batteries. J Mater Chem A, 2016, 4: 8091-8096

44 Dong A, Chen J, Vora PM, et al. Binary nanocrystal superlattice membranes self-assembled at the liquid-air interface. Nature, 2010, 466: 474-477

45 Wei J, Schaeffer N, Pileni MP. Solvent-mediated crystallization of nanocrystal 3D assemblies of silver nanocrystals: unexpected superlattice ripening. Chem Mater, 2016, 28: 293-302

46 Ha M, Kim JH, You M, et al. Multicomponent plasmonic nanoparticles: from heterostructured nanoparticles to colloidal composite nanostructures. Chem Rev, 2019, 119: 12208-12278

47 Zhang X, Lv L, Ji L, et al. Self-assembly of one-dimensional nanocrystal superlattice chains mediated by molecular clusters. J Am Chem Soc, 2016, 138: 3290-3293

48 Li X, Iqbal MA, Xu M, et al. $\mathrm{Au} @ \mathrm{Hg}_{x} \mathrm{Cd}_{1-x} \mathrm{Te}$ core@shell nanorods by sequential aqueous cation exchange for near-infrared photodetectors. Nano Energy, 2019, 57: 57-65

49 Cheng X, Sun R, Yin L, et al. Light-triggered assembly of gold nanoparticles for photothermal therapy and photoacoustic imaging of tumors in vivo. Adv Mater, 2017, 29: 1604894

50 Bayrak T, Helmi S, Ye J, et al. DNA-mold templated assembly of conductive gold nanowires. Nano Lett, 2018, 18: 2116-2123

51 Fink J, Kiely CJ, Bethell D, et al. Self-organization of nanosized gold particles. Chem Mater, 1998, 10: 922-926

52 Castelli A, de Graaf J, Marras S, et al. Understanding and tailoring ligand interactions in the self-assembly of branched colloidal nanocrystals into planar superlattices. Nat Commun, 2018, 9: 1-9

Acknowledgements This work was supported by the National Natural Science Foundation of China (NSFC, 51872030, 51631001, 21801015, 21643003, 51702016, 51501010 and 21703219), the Fundamental Research Funds for the Central Universities, Beijing Institute of Technology Research Fund Program for Young Scholars and Joint R\&D Plan of HongKong, Macao, Taiwan and Beijing (Z191100001619002). The authors acknowledge the critical testing work supported by Beijing Zhongkebaice Technology Service Co., Ltd.

Author contributions Chang Y performed the experiments assisted by Huang L and Pan R, and wrote the draft of manuscript. Liu J, Liu JJ, Rong $\mathrm{H}$ and Chen $\mathrm{W}$ supervised this study. Xu M provided suggestions and comments on the manuscript. Zhang J proposed the project and revised the manuscript. All authors contributed to the general discussion.
Conflict of interest The authors declare no conflict of interest.

Supplementary information Supporting data are available in the online version of the paper.

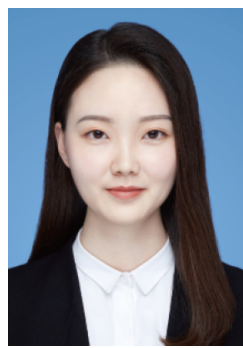

Yu Chang received her BSc degree from Zhengzhou University, China. She is currently a master degree candidate under the supervision of Prof. Jiatao Zhang in Beijing Institute of Technology (BIT), China. Her main research interests include the synthesis of Au@semiconductor core-shell hetero-nanocrystals and self-assembly of nanocrystals.

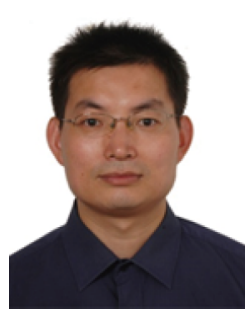

Jiatao Zhang was born in 1975. He earned his PhD from the Department of Chemistry, Tsinghua University, in 2006. Currently he is Xu Teli Professor in the School of Materials and Engineering, BIT. He was awarded the Excellent Young Scientist foundation of NSFC in 2013. He also serves as the director of Beijing Key Laboratory of Construction-Tailorable Advanced Functional Materials and Green Applications. His current research interest is inorganic chemistry of semiconductor-based hybrid nanostructures with novel optical, electronic properties for the applications in energy conversion and storage, catalysis, optoelectronics and biology.

\section{零维纳米晶体自组装形成微米级二维准纳米片: 从单组分到多组分组装单元}

常瑜, 徐萌, 黄柳, 潘容容, 刘佳佳, 刘佳, 我宏盼, 陈文星, 张加涛"

摘要 胶体纳米晶 $(\mathrm{NCs})$ 具有优良的性能和广阔的应用前景, 将其 自下而上自组装形成结构复杂且可控的大面积超晶格是一个重要 的研究方向. 等离子体纳米晶之间可控的间隙有益于电场增强, 因 此常常被用作组装单元来构建长程有序的大面积超结构, 尤其是 单层纳米晶的大规模组装. 本文采用溶剂挥发诱导气-液界面自组 装工艺, 成功得到了由单组分 $\mathrm{Au}$ 纳米晶和多组分 $\mathrm{Au} @ \mathrm{ZnS}$ 核壳异 质纳米晶(HNCs) 自组装形成的大面积二维准纳米片 (QNS). 整个 自组装过程在气-液界面上进行, 通过改变环境温度和胶体纳米晶 溶液的浓度来对自组装过程进行调控, 即通过精确控制溶剂的挥 发速率和胶体纳米晶浓度, 可以获得不同层数、不同堆积模式的 大面积二维准纳米片. 